\title{
Mortalidade neonatal de residentes em localidade urbana da região sul do Brasil*
}

\author{
Neonatal mortality in residents of a locality in southern Brasil
}

\author{
Luciana O. Bercini"*
}

\begin{abstract}
BERCINI, L.O. Mortalidade neonatal de residentes em localidade urbana da região sul do Brasil. Rev. Saúde Pública, 28:38-45, 1994. Caracterizou-se a mortalidade neonatal de residentes em Maringá Paraná, no ano de 1990, segundo algumas variáveis. O material de estudo constituiu-se de 87 óbitos. Foram utilizadas informaçóes retiradas das declarações de óbito, dos prontuários hospitalares, das entrevistas domiciliares, dos prontuários dos núcleos integrados de saúde e dos laudos de necrópsia. $O$ coeficiente de mortalidade neonatal foi de 19,4 por 1.000 nascidos vivos. A maioria dos óbitos ocorreu na primeira semana de vida $(92,0 \%)$, em recém-nascidos com menos de 37 semanas de gestação $(79,8 \%)$, em crianças com baixo peso ao nascer $(74,1 \%)$, em recém-nascidos do sexo masculino $(56,3 \%)$ e em crianças nascidas através de cesariana $(54,2 \%)$. As causas básicas de óbito foram codificadas de acordo com a Classificação Internacional das Doenças - 92 revisão. As Causas perinatais juntamente com as Anomalias congênitas responderam por $94,0 \%$ dos óbitos neonatais. As Causas perinatais foram responsáveis por $83,2 \%$ dos óbitos neonatais, onde a prematuridade ocupou o primeiro lugar, causando $15,8 \%$ das mortes. Constatou-se, também, que a prematuridade foi a causa associada mais freqüente dos óbitos de recém-nascidos $(59,0 \%)$.
\end{abstract}

Descritores: Mortalidade neonatal. Causa da morte. Fatores de risco.

\section{Introdução}

A mortalidade infantil reflete as condições de vida e de saúde das populações e o seu coeficiente é utilizado como indicador do nível de desenvolvimento e da qualidade de vida das nações.

A divisão da mortalidade infantil em neonatal e pós-neonatal é feita no sentido de se avaliar indiretamente a importância das causas endógenas e exógenas de óbito. As primeiras estão relacionadas às condições desfavoráveis do recém-nascido, tais como imaturidade e anomalias congênitas. Já as segundas são o produto da atuação de condições ambientais hostis sobre crianças nascidas em boas condições. Enquanto as endógenas são de difícil redução, implicando conhecimentos e técnicas mais sofisticadas, as exógenas são dependentes de melhoria das condições gerais de vida e das intervençōes específicas de caráter médico-sanitário ${ }^{15}$.

\footnotetext{
* Baseado em Dissertação de Mestrado apresentada na Escola Paulista de Medicina, em 1993.

* Departamento de Enfermagem da Universidade Estadual de Maringá - Maringá, PR - Brasil

Separatas/Reprints: L.O. Bercini - Av. Colombo, $3690-80020-900$ - Maringá, PR - Brasil
}

Os componentes neonatal e pós-neonatal apresentam comportamentos distintos, em áreas com mortalidade infantil baixa e alta. Observa-se que, no primeiro caso, o coeficiente é quase totalmente representado pela mortalidade neonatal $(60$ a $80 \%)$ e, no segundo caso, a taxa é quase totalmente representado pela mortalidade pós-neonatal. Quando a melhoria das condições ambientais vão permitindo reduzir a mortalidade infantil, verifica-se que essa redução se faz às custas da pós-neonatal. Mais tarde, consegue-se diminuir a neonatal, atuando na assistência pré, trans e pós-natais ${ }^{11}$.

Os estudos de mortalidade são feitos, em geral, através dos dados das declarações de óbito (DO), que se constitui instrumento muito importante para obtenção de variáveis utilizadas para caracterizar os óbitos.

O uso dos dados de mortalidade oriundos das DO levanta questōes sobre a confiabilidade e a validade das informações nela contida ${ }^{25}$.

Muitos esforços têm sido empreendidos no sentido de alertar sobre a importância do preenchimento adequado da $\mathrm{DO} \mathrm{O}^{9,13,14}$, mas o que se verifica, na maioria das vezes, é que esta é vista somente como uma exigência legal para o sepultamento e não como uma possivel fonte geradora de dados 
sobre a saúde de determinada população.

Os estudos de mortalidade que procuram completar os dados das DO com informaçōes retiradas dos prontuários hospitalares e com entrevistas nos domicilios daqueles que foram a óbito, permitem uma análise aprofundada das características dos óbitos, principalmente em relação à causa básica e associada de morte ${ }^{1,11,22}$.

Vários fatores necessitam ser estudados para se conhecer mais profundamente a mortalidade neonatal. $\mathrm{O}$ conhecimento das inter-relações e associações dos fatores maternos, fetais e do parto constituem campo interessante para análise; esses fatores devem ser considerados e combinados com as causas básicas e associadas de morte ${ }^{24}$.

O presente trabalho tem como objetivo caracterizar a mortalidade neonatal, segundo algumas variáveis. Para tanto, foram utilizados dados relativos a residentes de Maringá, Estado do Paraná, no ano de 1990.

\section{Metodologia}

O material de estudo constituiu-se de 87 óbitos, correspondendo a todas as crianças menores de 28 dias de idade falecidas no período de $1^{2}$ de janeiro a 31 de dezembro de 1990 e residentes no Município de Maringá, localizado na região norte do Estado do Paraná, a $428 \mathrm{~km}$ ao noroeste de Curitiba, capital do Estado. Tem uma população de 239.930 habitantes, de acordo com os dados do Censo ${ }^{6}$ de 1991 , e $473 \mathrm{~km}^{2}$ de superfície ${ }^{20}$.

Criado em 1947, o Município de Maringá representa um dos três pólos de desenvolvimento social e econômico mais importante do Estado do Paraná. A cidade teve um grande crescimento populacional, social e econômico em conseqüência do surto cafeeiro ${ }^{20}$. Atualmente, superado o "ciclo do café", existe uma diversificação da produção agrícola local e regional, compreendendo outras culturas como soja, milho, algodão, cana-de açúcar, trigo e uva. Essa mudança foi responsável pelo desenvolvimento da indústria da região, ocasionando alteração radical no panorama rural da cidade, como ficou evidenciado pelos dados do Censo ${ }^{5}$ de 1980 onde apenas $4,5 \%$ da população residia na zona rural.

Para realização do estudo adotou-se a metodologia adaptada daquela utilizada por Puffer \& Serrano $^{22}$ (1973), na "Investigação Interamericana de
Mortalidade na Infância".

Os dados do estudo teve origem nas DO de onde foram obtidos a identificação da criança e o local de ocorrência do evento. A partir dessas informações foram feitas consultas aos prontuários hospitalares. Nos casos de óbitos ocorridos no domicilio e em hospitais de outros municípios, e para aqueles cujos prontuários não foram localizados, foram realizadas entrevistas domiciliares. Nos casos de morte atestada pelo Instituto Médico Legal (IMI), foram realizadas consultas aos laudos das necrópsias. Foram feitas, também, consultas aos prontuários dos Núcleos Integrados de Saúde (NIS), que são os postos de saúde municipais.

Em relação aos prontuários hospitalares, dificultaram a realização do levantamento fatores como: letra ilegível, descrição sumária do caso e pouca informação sobre a gestação, o parto e as condições de nascimento da criança.

Alguns dados não puderam ser coletados por falta de anotação no prontuário, o que determinou a utilização da categoria ignorado em quase todas as variáveis.

Situação similar também foi evidenciada na utilização dos prontuários dos NIS, pois somente em alguns postos de saúde havia o preenchimento completo da ficha de atendimento pré-natal; na maioria eram feitas anotações sumárias no prontuário da cliente.

As causas básica e associada foram obtidas através da análise das informações do prontuário hospitalar, do prontuário do NIS, da entrevista domiciliar, do laudo do IML e, em alguns casos, da colaboração de médicos neonatologistas. As causas foram codificadas conforme a lista tabular da Classificação Internacional de Doenças (CID) - 9: Revisão, $1975^{18}$.

\section{Resultados e Discussão}

Coeficiente de mortalidade - O coeficiente de mortalidade infantil encontrado para o município foi de $25,6 \%$ n.v., sendo a taxa de mortalidade neonatal de $19,4 \%$ n.v., representando $75,7 \%$ da mortalidade infantil, o que mostrou o predomínio do componente neonatal nos óbitos de menores de um ano.

O fato da mortalidade neonatal ser superior à pós-neonatal está de acordo com o nível relativa- 
mente baixo de mortalidade, uma vez que a queda do coeficiente de mortalidade infantil em paises em desenvolvimento ocorre principalmente às custas da mortalidade pós-neonatal, com uma menor redução do componente neonatal, que passa a ser proporcionalmente maior ${ }^{1,17,27}$. A situação difere nos países desenvolvidos, como Estados Unidos da América (EUA), Finlândia, Inglaterra e País de Gales, onde o peso relativo das doenças infecciosas é baixo e a diminuição se faz principalmente devido à redução da mortalidade neonatal ${ }^{17,19}$.

A mortalidade neonatal está vinculada a fatores biológicos e de Assistência intra-hospitalar. Portanto, sua redução é de custo elevado e está ligada a investimentos consideráveis destinados a fortalecer os serviços hospitalares de alta complexidade. Porém, em muitos países em desenvolvimento ainda não se utiliza suficientemente a tecnologia básica preventiva que reduz a mortalidade neonatal ${ }^{17}$.

O coeficiente de mortalidade neonatal precoce (menores de sete dias de vida) foi de $17,8 \%$ n.v. e o de neonatal tardio (de sete a 28 dias de vida) foi de $1,6 \%$ n.v. No desmembramento do coeficiente de mortalidade neonatal precoce obteve-se a taxa de mortalidade no primeiro dia de vida de $10,5 \%$ n.v.

Idade - Os óbitos ocorridos nas primeiras $24 \mathrm{~h}$ de vida representaram $54,0 \%$ dos óbitos neonatais. Compreenderam $92,0 \%$ das mortes de menores de 28 dias (Tabela 1), os óbitos neonatais precoces.

Resultados semelhantes foram obtidos por Moscona e col. ${ }^{16}$ (1985), em estudo sobre mortalidade neonatal na cidade de Buenos Aires, Argentina, onde os óbitos neonatais precoces foram responsáveis por $83,25 \%$ dos óbitos neonatais e as mortes ocorridas nas primeiras 24 h de vida por 44,8\%; e por Victora e col. ${ }^{27}$ (1989), em Pelotas, Rio Grande do Sul, onde os óbitos ocorridos na primeira semana de vida foram responsáveis por $81,5 \%$ dos óbitos neonatais e por $51,2 \%$, no primeiro dia de vida.

A mortalidade na primeira semana de vida constitui o componente da mortalidade infantil mais difícil de reduzir, e sua diminuição, assim como a das seqüelas invalidantes, que se originam nesse período, depende de novas estratégias no campo perinatal com ações dirigidas ao desenvolvimento e fortalecimento dos serviços de saúde materno-infantis ${ }^{17}$.

Sexo - Dos 87 óbitos neonatais, 56,3\% foram de crianças do sexo masculino e $43,7 \%$ do sexo feminino.

Moscona e col. ${ }^{16}$ (1985) encontraram $54,1 \%$ de óbitos de recém-nascidos (RN) do sexo masculino e 45,9\% do feminino. Em Botucatu - São Paulo, $65,2 \%$ dos óbitos neonatais foram do sexo masculino e $34,8 \%$ do feminino ${ }^{12}$. Estes percentuais foram semelhantes aos encontrados no presente estudo.

Laurenti e col. ${ }^{10}$ (1985) afirmaram que em relação à mortalidade segundo o sexo, é fato comum de observação que, sempre e em qualquer área, o coeficiente de mortalidade do sexo masculino é maior que o do feminino, em todas as idades. Referiram ainda que é gerado maior número de indivíduos do sexo masculino do que do feminino. Nos países desenvolvidos nascem 5 a $6 \%$ mais meninos do que meninas, entretanto, os paises em desenvolvimento não seguem esse padrão. Tal fato é decorrente do grande número de perdas fetais e, nestas, a fragilidade masculina pode ser destacada; ela será tanto maior quanto mais precoce for a perda. Ainda assim, nesses paises, nascem mais meninos do que meninas.

Duração da gestação - A maioria (79,8\%) dos óbitos neonatais, ocorreu em RN com menos de 37 semanas de gestação, revelando a relação entre mortalidade neonatal e RN pré-termo (Tabela 2).

A duração da gestação foi inferior a 37 semanas em todos os óbitos neonatais cuja gravidez foi dupla (12 casos), mostrando a relação entre mortalidade neonatal, gemelaridade e parto prematuro.

Guaschino e col. ${ }^{17}$ (1986) afirmaram que a complicação típica da gravidez dupla é o parto prematuro. Em estudo realizado em Pavia - Itália, em 1973/83, com 154 gestações duplas, os autores encontraram intensa influência de idade gestacional, menos de 34 semanas de duração, com mortalidade perinatal e neonatal. Não encontraram diferença significante na mortalidade neonatal com ida-

Tabela 1. Distribuição dos óbitos neonatais segundo idade, Maringá, PR, 1990.

\begin{tabular}{lcc}
\hline \multicolumn{1}{c}{ Idade (dias) } & $\mathrm{N}^{2}$ & $\%$ \\
\hline $0 \vdash 1$ & 47 & 54,0 \\
$1 \vdash 7$ & 33 & 38,0 \\
Subtotal $(0 \vdash 7)$ & 80 & 92,0 \\
$7 \vdash 28$ & 07 & 8,0 \\
\hline Total & 87 & 100,0 \\
\hline
\end{tabular}


Tabela 2. Distribuição dos óbitos neonatais, segundo duração da gestação, Maringá, PR, 1990.

\begin{tabular}{lcc}
\hline Duracăo da gestaçăo (semanas) & $\mathrm{N}^{2}$ & $\%\left(^{*}\right)$ \\
\hline$<37$ & 67 & 79,8 \\
$\geq 37$ & 17 & 20,2 \\
lgnorado & 03 &.. \\
\hline Total & 87 & 100,0 \\
\hline
\end{tabular}

(") Os percentuais foram calculados sobre o total de casos com informaçäo conhecida.

de gestacional, entre 34 e 37 semanas de duração, em comparação com os $\mathrm{RN}$ a termo.

Tipo de parto - A cesariana foi realizada em $54,2 \%$ dos óbitos e o parto normal ocorreu em $42,2 \%$ dos casos com informação conhecida.

Segundo Instituto Nacional de Assistência Médica da Previdência Social (INAMPS) ${ }^{8}$, verifica-se percentual elevado $(59,6 \%)$ de cesarianas em gestantes de Maringá, em 1990. Provavelmente esse percentual foi maior, já que não foram incluídas as cesarianas de caráter particular e de medicina de grupo, correspondendo somente às realizadas através daquele Instituto. A clínica obstétrica atualmente aceita niveis de até $20,0 \%{ }^{26}$, o que mostra que grande parte das cesarianas realizadas no município estudado provavelmente não tiveram indicação obstétrica.

Em $68,9 \%$ dos casos, nos quais a criança nasceu através de cesariana, havia informação no prontuário hospitalar ou na entrevista domiciliar sobre a necessidade da realização desse procedimento. Portanto, em $31,1 \%$ dos casos não se obteve a informação sobre a indicação obstétrica de cesariana, o que pode indicar excesso na sua realização resultando em uma situação de risco tanto para a mãe quanto para o RN.

Assistência pré-natal -Em 57,5\% dos prontuários hospitalares não se obteve informação sobre a assistência pré-natal; conseqüentemente, menos da metade dos prontuários das crianças que foram a óbito possuía alguma informação sobre o pré-natal realizado pela mãe.

Esses dados revelaram a falta de integração entre os organismos que realizam o pré-natal e a assistência ao parto. Tanaka e col. ${ }^{26}$ (1989) afirmaram que esta falta de integração impede que o processo reprodutivo seja visto em sua totalidade, favorecendo a descontinuidade na assistência à saúde do binômio mãe-filho, culminando com maior risco de morte da mulher e da criança. Os autores referiram ainda que a integração entre $o$ atendimento pré-natal, a assistência ao parto e ao $\mathrm{RN}$ deve ocorrer para que a qualidade da assistência seja garantida.

Em 5,4\% dos casos com informação conheci$\mathrm{da}$, a mãe não realizou o pré-natal. Entre as que o realizaram, a assistência foi feita em $57,1 \%$ pelo Sistema Único de Saúde, em $8,6 \%$ por medicina de grupo, em $8,6 \%$ por particular e em $25,7 \%$ dos casos foi ignorado. Estes corresponderam a aqueles cuja única informação registrada no prontuário era de que a assistência pré-natal havia sido realizada.

Idade materna - Em 77,4\% dos óbitos neonatais as mães tinham menos de 30 anos de idade. Do total, 22,6\% eram menores de 20 anos de idade. A mãe mais jovem tinha 13 anos e teve gêmeos que foram a óbito. Duas mães tinham 40 anos e foram as mais idosas do estudo.

Segundo a OPAS ${ }^{17}$ (1990), a maior contribuição à fecundidade provém de mulheres com idade entre 20 anos a 34 anos, sendo que os grupos etários extremos (menores de 20 e maiores de 35 anos de idade) constituem grupos de alto risco de mortalidade materna e neonatal.

Friede e col. ${ }^{4}$ (1987) encontraram nos nascimentos ocorridos em 1980, nos EUA, uma forte associação entre a baixa idade materna e o aumento do risco de morte infantil. Recém-nascidos de mães adolescentes tiveram um risco de mortalidade 1,5 a 3,5 vezes maior do que comparados com aqueles nascidos de mães com idade entre 25 a 29 anos.

Peso ao nascer - Os óbitos de menores de 28 dias concentraram-se principalmente no grupo de baixo peso ao nascer (inferior a $2.500 \mathrm{~g}$ ), representando $74,1 \%$ dos casos, o que confirma a conhecida relação do baixo peso ao nascer e mortalidade neonatal. Em 39,5\% dos óbitos neonatais as crianças nasceram com extremo baixo de peso (inferior a $1.500 \mathrm{~g})$. Um RN apresentou peso excessivo (5.000g ou mais) (Tabela 3 ).

Em Maringá obteve-se um percentual de óbitos de $\mathrm{RN}$ com peso ao nascer inferior a $2.500 \mathrm{~g}$ comparável ao dos EUA, onde $2 / 3$ das mortes no período neonatal ocorrem em crianças com baixo peso ao nascer ${ }^{21}$. Moscona e col. ${ }^{16}$ (1985) identifi- 
Tabela 3. Distribuição de óbitos neonatais, segundo peso ao nascer, Maringá, PR, 1990.

\begin{tabular}{lcc}
\hline Peso ao nascer (gramas) & № & $\%\left(^{\star}\right)$ \\
\hline$<1.500$ & 32 & 39,5 \\
$1.500+2.500$ & 28 & 34,6 \\
Subtotal $(<2.500)$ & 60 & 74,1 \\
$\geq 2.500$ & 21 & 25,9 \\
Ignorado & 06 &.. \\
\hline Total & 87 & 100,0 \\
\hline
\end{tabular}

(*) Os percentuais foram calculados sobre o total dos casos com informação conhecida.

caram que $68,1 \%$ dos óbitos neonatais ocorreram em RN com baixo peso ao nascer, e que $43,9 \%$ das mortes neonatais foram de crianças com peso inferior a $1.500 \mathrm{~g}$ resultados estes semelhantes aos do presente estudo.

Somando as crianças de baixo peso ao nascer com aquelas de peso deficiente $(2.500 \vdash 3.000 \mathrm{~g})$, assim considerado por Puffer \& Serrano ${ }^{23}$ (1988), foram obtidos $85,2 \%$ dos óbitos. Para os citados autores, as crianças nascidas com peso deficiente representam uma proporção considerável dos nascidos vivos em alguns paises; e todos os RN com peso inferior a $3.000 \mathrm{~g}$ têm um grande risco de mortalidade e morbidade no primeiro ano de vida.

Verificou-se que a totalidade dos óbitos neonatais de gestações duplas ( 12 casos) foram de $\mathrm{RN}$ de baixo peso, mostrando a relação entre mortalidade neonatal, gemelaridade e baixo peso ao nascer.

Dos óbitos ocorridos na primeira semana de vida, $74,7 \%$ foram de crianças com baixo peso ao nascer e $40,0 \%$ foram de $\mathrm{RN}$ com extremo baixo peso, demonstrando a relação entre a mortalidade neonatal precoce e o baixo peso ao nascer. Dos óbitos ocorridos no primeiro dia de vida, $60,0 \%$ foram de crianças com extremo baixo peso ao nascer.

Moscona e col. ${ }^{16}$ (1985) encontraram que dos óbitos ocorridos na primeira semana de vida, $78,9 \%$ foram de crianças com baixo peso ao nascer e que das mortes ocorridas no primeiro dia de vida, $59,0 \%$ foram de crianças com extremo baixo peso ao nascer. Esses resultados estão compativeis com os do presente estudo.

Como era esperado, dos óbitos de crianças com baixo peso ao nascer, a maior parte $(95,0 \%)$ foi de RN com menos de 37 semanas de gestação, revelando a relação da mortalidade neonatal com crianças com baixo peso ao nascer e pré-termo, sendo a prematuridade uma causa importante de baixo peso. Verificou-se retardo do crescimento intra-uterino em $5,0 \%$ dos óbitos, pois ocorreram em $\mathrm{RN}$ a termo e com baixo peso ao nascer ${ }^{23}$.

O retardo no crescimento intra-uterino relaciona-se com vários fatores, dos quais destacam-se principalmente os maternos, tais como: peso deficiente antes da gravidez, pouco ganho ponderal durante a gestação, infecções das vias urinárias, ação sinérgica das infecções e da deficiência nutricional maternas e pré-eclâmpsia ${ }^{23}$.

Local de ocorrência do óbito - Como mais de $50 \%$ dos óbitos neonatais ocorreram no primeiro dia de vida e cerca de $90 \%$, na primeira semana, a grande maioria dos óbitos neonatais $(96,6 \%)$ ocorreu no hospital. Percentual semelhante foi observado na Investigação Interamericana ${ }^{22}$, nos projetos da América do Norte, onde $97,5 \%$ dos óbitos neonatais ocorreram no hospital. O percentual encontrado foi um pouco superior ao dos projetos latinoamericanos da referida investigação, onde $82,1 \%$ dos óbitos de menores de 28 dias ocorreram no hospital.

Dos óbitos neonatais, $90,9 \%$ ocorreram em hospitais de Maringá, 5,7\% em hospitais de outros municipios e $3,4 \%$ no domicílio.

Causa básica de óbito - Os dois principais grupos de causas básicas de óbito, por ordem de ocorrência, foram: causas perinatais $(83,2 \%)$ e anomalias congênitas $(10,8 \%)$. Carvalho e col. ${ }^{3}$ (1990), na região metropolitana do Rio de Janeiro, encontrou $83,4 \%$ de causas perinatais entre os óbitos neonatais. Um percentual menor foi achado por Buehler e col. ${ }^{2}$ (1987), nos EUA, onde 62,1\% das mortes neonatais, de RN de gestação única, foram atribuídas às causas perinatais; e pela "Investigação Interamericana", onde cerca de $50,0 \%$ dos óbitos de menores de 28 dias foram devido a essas causas, no total dos projetos estudados 22 . Quanto às anomalias congênitas, Buehler e col. ${ }^{2}$ (1987) observaram um percentual maior nesse grupo de causa, sendo responsável por $27,1 \%$ dos óbitos neonatais, de $\mathrm{RN}$ de gestação única. Moscona e col. ${ }^{16}$ (1985) encontraram um percentual de $12,2 \%$, semelhante ao identificado no presente estudo. 
Considerando-se os três dígitos, a prematuridade (CID 765) apareceu em primeiro lugar, sendo responsável por $15,8 \%$ dos óbitos neonatais, seguida da hipóxia intra-uterina e asfixia ao nascer (CID 768), respondendo por $14,5 \%$ das mortes, e das complicações da placenta, do cordão umbilical ou das membranas (CID 762) causadoras de $13,3 \%$ dos óbitos de menores de 28 dias de vida.

Somando-se as causas perinatais com as anomalias congênitas temos $94,0 \%$ dos óbitos de menores de 28 dias, o que mostrou a importância dessas causas na mortalidade neonatal, em Maringá. Os quatro grupos de causas restantes, doenças infecciosas e parasitárias, doenças do sangue e dos órgãos hematopoéticos, mal definidas e externas, foram responsáveis por apenas $6,0 \%$ dos óbitos neonatais.

Verificou-se que as causas perinatais foram as mais importantes em todos os grupos etários, principalmente na primeira semana de vida, causando 79,5\% das mortes neonatais (Tabela 4). Na Investigação Interamericana também observou-se que este grupo de causas desempenhou papel determinante na mortalidade no periodo neonatal precoce ${ }^{22}$.

Os óbitos devido às causas perinatais diminuíram na medida em que houve o aumento da idade; ao contrário, os óbitos por anomalias congênitas aumentaram na medida em que houve o aumento da idade (Tabela 4).

A Tabela 4 mostra também que as causas perinatais e as anomalias congênitas foram responsá- veis por todas as mortes ocorridas nas primeiras 24 $h$ de vida.

O coeficiente de mortalidade neonatal por causas perinatais foi de $15,4 \%$ n.v., demonstrando a relevância desse grupo de causas no município. Esse coeficiente foi semelhante ao identificado por Victora e col. ${ }^{27}$ (1989), que foi de 16,0\%o n.v.; e superior à taxa da Inglaterra e País de Gales ${ }^{19}$, que foi de $3,2 \%$ n.v.

O coeficiente de mortalidade neonatal por anomalias congênitas, no presente estudo, foi de $2,0 \%$ n.v., sendo menor que o identificado por Victora e col. $^{27}$ (1989) de 3,2\%o n.v. e semelhante ao coeficiente da Inglaterra e Pais de Gales de 1,7\%o n.v. ${ }^{19}$ Este coeficiente também foi menor que o observado em Sherbrooke - Canadá (3,8\% n.v.), o qual apresentou uma alta freqüência de anomalias graves do sistema nervoso central ${ }^{22}$. O coeficiente encontrado pode estar indicando uma insuficiência de diagnóstico em Maringá, devido a falta de recursos.

Para Puffer \& Serrano 22 (1973), a integridade da informação sobre as anomalias congênitas está na dependência da evidência diagnóstica disponível. A freqüência dos diagnósticos depende, em parte, da gravidade das anomalias e também dos sinais visiveis, portanto é provável que as anomalias graves do sistema nervoso foram sempre diagnosticadas. Porém certas anomalias congênitas do coração e do aparelho circulatório podem passar despercebidas ou não ser claramente especificadas na ausência de um exame pós-morte.

Tabela 4. Distribuição de óbitos neonatais, segundo causas básicas e idade, Maringá, PR, 1990.

\begin{tabular}{|c|c|c|c|c|c|c|c|c|}
\hline \multirow{3}{*}{ Causas básicas de óbito (") } & \multicolumn{6}{|c|}{ Idade (dias) } & & \\
\hline & \multicolumn{2}{|c|}{$0 \vdash 1$} & \multicolumn{2}{|c|}{$1 \vdash 7$} & \multicolumn{2}{|c|}{$7 \vdash 28$} & \multicolumn{2}{|c|}{ Total } \\
\hline & Ne & $\%^{(+)}$ & $\mathrm{N}^{2}$ & $\%(+)$ & Ne & $\%\left({ }^{-\infty}\right)$ & № & $\%\left({ }^{(*)}\right.$ \\
\hline Doenças infecciosas e parasitárias & - & - & 2 & 6,5 & - & - & 2 & 2,4 \\
\hline Doenças do sangue-órgãos hematopoéticos & - & - & - & - & 1 & 14,3 & 1 & 1,2 \\
\hline Anomalias congênitas & 1 & 2,2 & 6 & 19,4 & 2 & 28,6 & 9 & 10,8 \\
\hline Perinatais & 44 & 97,8 & 22 & 70,9 & 3 & 42,8 & 69 & 83,2 \\
\hline Mal definidas & - & - & 1 & 3,2 & - & - & 1 & 1,2 \\
\hline Externas & - & - & - & - & 1 & 14,3 & 1 & 1,2 \\
\hline Ignorada & 2 & - & 2 & - & - & - & 4 & - \\
\hline Total & 47 & 100,0 & 33 & 100,0 & 7 & 100,0 & 87 & 100,0 \\
\hline
\end{tabular}

(") CID - gr revisão $0^{18}$

("*) Os percentuais foram calculados sobre total dos casos com inforamção conhecida. 
Tabela 5. Distribuição dos óbitos neonatais, segundo as principais causas básicas e associadas, Maringá, PR, 1990.

\begin{tabular}{|c|c|c|c|c|}
\hline \multirow{2}{*}{ Causas de óbito (") } & \multicolumn{2}{|c|}{ Básica } & \multicolumn{2}{|c|}{ Associada } \\
\hline & $N^{2}$ & $\%\left({ }^{+*}\right)$ & $N^{9}$ & $\%(*+)$ \\
\hline Complicaçōes maternas da gravidez (761) & 7 & 8,4 & 16 & 19,3 \\
\hline Complicaçōes placenta, cordāo umbilical ou membranas (762) & 11 & 13,3 & 2 & 2,4 \\
\hline Prematuridade (765) & 13 & 15,8 & 49 & 59,0 \\
\hline Hipóxia intra-uterina asfixia ao nascer (768) & 12 & 14,5 & 20 & 22,9 \\
\hline Outras causas & 40 & 48,0 & 9 & 10,8 \\
\hline Ignorado & 4 & - & - & - \\
\hline Total & 87 & 100,0 & 94 & - \\
\hline
\end{tabular}

(") CID - $9^{9}$ revisão ${ }^{18}$

(*) Os percentuais foram calculados sobre o total dos casos com informação conhecida.

O grupo de causas denominado outras anomalias congênitas do coração, sem especificação (CID 746.9), foi o mais freqüente, sendo responsável por $33,4 \%$ dos óbitos neonatais. A ausência da realização de necrópsia nestes $\mathrm{RN}$ provavelmente contribuiu para a não especificação dessas causas.

Causas associadas de óbito - A prematuridade (CID 765) foi a causa associada mais freqüente $(59,0 \%)$ dos óbitos neonatais, evidenciando-se assim o seu papel de destaque como a mais importante causa associada dos óbitos desse grupo etário (Tabela 5).

A prematuridade apareceu com maior freqüência como causa associada do que como causa básica (59,0\% e $15,8 \%$ respectivamente), já que as regras de classificação de causa básica especificam que não se deve usá-la se constar qualquer outra causa de mortalidade perinatal ${ }^{18}$.

Puffer \& Serrano 22 (1973) mostraram que a prematuridade constitui um problema muito grave em várias cidades da América Latina e a consideraram como o fator mais importante na vulnerabilidade à doença, aumentando o risco de morte no período neonatal. A prematuridade foi considerada como causa associada em $\mathbf{5 7 , 5 \%}$ dos óbitos neonatais, percentual este muito semelhante ao encontrado no presente estudo.

A prematuridade, como causa associada de óbito, esteve intimamente relacionada com a maioria das causas básicas, ou seja, na totalidade dos óbitos neonatais causados por afeç̧ões maternas, complicações maternas da gravidez e hipóxia intrauterina e asfixia ao nascer; e na maioria das mortes por complicações da placenta, do cordão umbilical ou das membranas, por sindrome de dificuldade respiratória e por outras causas perinatais apresentaram associação com prematuridade.

$\mathrm{Na}$ "Investigação Interamericana"22 também observou-se forte associação da prematuridade com as referidas causas básicas, sendo que a prematuridade foi classificada como uma conseqüencia das afecções maternas, das complicações maternas da gravidez e do descolamento prematuro da placenta; e como causa contribuinte da hipóxia intra-uterina e asfixia ao nascer e das afecções do cordão umbilical.

\section{Agradecimento}

A Prof ${ }^{2} D r^{2}$ Lucila Amaral Carneiro Vianna, do Departamento de Enfermagem da Escola Paulista de Medicina, pela orientação e colaboração.

BERCINI, L.O. [Neonatal mortality in residents of a locality in southem Brazil]. Rev. Saúde Pública, 28:38-45, 1994. Neonatal mortality rates in Maringá, Paraná State, Brazil, in 1990 are characterized in terms of several variables. Information concerning these events was provided by death certificates, hospital archives, home interviews, health center files and necropsy records. Neonatal mortality rates were 19.4 per 1,000 live births. Most of the deaths occurred: the first week of life $(92.0 \%)$; newborns with less than 37 weeks of gestation $(79.8 \%)$; low birth weight infants (74.1\%); male newborns (56.3\%); and cesarean section births $(54.2 \%)$. The underlying causes of death were codified in accordance with the "International Diseases Classification" $-9^{\text {th }}$ revision. Perinatal Causes together with Congenital Anomalies accounted for $94.0 \%$ of these deaths in addition also to being responsible for $83.2 \%$ of neonatal deaths; prematurity was considered the main cause, 
accounting for $15.8 \%$ of deats while being the most frequent cause associated with newborn deats $(59.0 \%)$.

Keywords: Neonatal mortality. Cause of death. Risk factors.

\section{Referência Bibliográficas}

1. AMIGO, $\mathrm{H}$. et al. Mortalidade em menores de cinco anos na cidade de Recife, PE (Brasil). Tendenncias e associações. Rev. Saúde Pública, 19:531-42, 1985.

2. BUEHLER, J.W. et al. Birth weigth-specific causes of infant mortality, United States, 1980. Public Health Rep., 102 (suppl. 2):162-71, 1987.

3. CARVALHO, M.L. et al. Concordância na determinação da causa básica de óbito em menores de um ano na região metropolitana do Rio de Janeiro, 1986. Rev. Saúde Pública, 24 (suppl. 1):20-7, 1990.

4. FRIEDE, A. et al. Young matemal age and infant mortality: the role of low birth weigth. Public Health Rep., 102 (Suppl. 2):192-9, 1987.

5. FUNDAÇĀO IBGE Censo Demográfico do Paraná. Rio de Janeiro, 1982. v.1. (9 Recenseamento Geral do Brasil, 1980).

6. FUNDAÇĀO IBGE População dos municipios do Estado do Paraná. Censo Demográfico de 1991. Dados preliminares. Curitiba, 1992.

7. GUASCHINO, S. et al. Assessment of peri-neonatal mortality and morbidity risk in twin pregnancy. Clin. Exp. Obstet. Gynecol., 13 (suppl. 1/2):18-25, 1986.

8. INSTITUTO NACIONAL DE ASSISTĖNCIA MÉDICA DA PREVIDÊNCIA SOCIAL. Boletim DATAPREV/Relaçäo dos procedimentos pagos. Autorização de Intenação Hospitalar, 1991.

9. LAURENTI, R. \& MELLO JORGE, M.H.P. $O$ atestado de óbito. 2 ed. São Paulo, Centro da OMS para Classificação de Doenças em Português, 1987. (Série Divulgação $n^{2} 1$ ).

10. LAURENTI, R. et al. Estatísticas de saúde. São Paulo, EPU/EDUSP, 1985.

11. LAURENTI, R et al. Mortalidade em mulheres em idade fértil no municipio de São Paulo (Brasil), 1986. I - Metodologia e resultados gerais. Rev. Saúde Pública, 24(suppl. 2):12833, 1990.

12. MACHARELLI, C.A. \& OliveiRA, L.R. Perfil do risco de óbito de crianças menores de um ano residentes em localidade do Estado de São Paulo, Brasil, 1987. Rev. Saúde Pública, 25(suppl. 2):121-8, 1991.

13. MELLO JORGE, M.H.P. Registro dos eventos vitais: sua importância em saúde pública. São Paulo, Centro da OMS para a Classificação de Doenças em Portuguès, 1990. (Série Divulgação, 5).

14. MINISTÉRIO DA SAÚDE. Secretaria Nacional de Açōes básicas de Saúde. Divisảo Nacional de Epidemiologia. Manual de instruçōes para o preenchimento da declara. ção de óbito. $2^{4}$ ed. Brasilia, Centro de Documentação, 1985. (Normas e Manuais Técnicos, 24).

15. MONTEIRO, C.A. et al. A mortalidade. In: Monteiro, C.A. Saúde e nutriçāo das crianças de São Paulo. São Paulo, HUCITEC/EDUSP, 1988. p.143-58.

16. MOSCONA, R.R. et al. Estudio de la mortalidad neonatal en la ciudad de Buenos Aires en 1984. Arch. Arg. Pediatr., 83(suppl. 6):307-12, 1985.

17. ORGANIZACION PARANAMERICANA DE LA SALUD. Las condiciones de salud en las Américas. Washington, 1990. v.1. p.55-71. (OPS - Publicación Científica, 524).

18. ORGANIZACION PANAMERICANA DE LA SALUD. Manual de la clasificación estadística internaciona de enfermedades, traumatismos y causas de defunción. 9' rev. 1975. Washington, 1978. v.1. (OPS - Publicación Cientifica, 353)

19. PHAROAH, P.O.D. \& ALBERMAN, E. Annual statistical review. Arch dis. Child., 63:1511-5, 1988.

20. PREFEITURA DO MUNICIPIO DE MARINGÁ. Perfilyplano municipal de desenvolvimento. Maringá, 1990.

21. PREVENTING low birthweigth. Washington, National Academy Press, 1985.

22. PUFFER, R.R. \& SERRANO, C.V. Características de la mortalidad en la niñez. Washington, 1973. (OPS - Publicación Cientifica, 262).

23. PUFFER, R.R. \& SERRANO, C.V. Características del peso al nacer. Washington, Organización Panamericana de la Salud, 1988. (OPS - Publicacion Cicntifica, 504)

24. PUFFER, R.R. \& SERRANO, C.V. Nucvos enfoques para los estudios epidemiológicos sobre estadísticas de mortalidad. Bol. Of. Sanit. Panam., 107(suppl. 4):277-95, 1989.

25. ROSENBERG, H.M. Improving cause-death statistics. [Editorial] Am. J. Public Heath, 79(suppl. 5):563-4, 1989.

26. TANAKA, A.C. d'A. et al. Situação de saúde manterna e perinatal no Estado de São Paulo, Brasil. Rev. Saúde Pública, 23(suppl. 1):67-75, 1989.

27. VICTORA, C.G. et al. Epidemiologia da desigualdade. 2 ed. São Paulo, HUCITEC, 1989.

Recebido para publicação em 26.7.1993 Aprovado para publicaçāo em 21.10.1993 